$-48.50^{\circ}$ (c, 0.545). Yield, $125 \mathrm{mg}$.

Anal. Found: C, 87.52; H. 12.28. Calcd. for $\mathrm{C}_{30} \mathrm{H}_{50}$ : C, $87.83 ; \mathrm{H}, 12.27$.

Its Infrared pattern agreed with that of isoilexene (Fig. Ie).

The mixture of this substance and isoilexene (m.p. $183-185^{\circ}$ ) melted at $181-182^{\circ}$, and it was found to be optically inactive.

Acknowledgements. The author wishes to express his sincere thanks to Prof. Dr. S. Iseda of this Laboratory, to Prof. Dr. Y. Oshima of
Kyushu University, and to Prof. Dr. E. Sebe of Kumamoto University, for their helpful advice and encouragement throughout the course of this work. $\mathrm{He}$ is also deeply indebted to Messrs. H. Kozuma and K. Kawasaki of the Technical Department of Minamata Factory, Shin Nippon Chisso Hiryo and Co., Minamata, for their kindness for measuring infrared spectra, and to Miss M. Kinoshita of Kumamoto University, for carrying out elementary analyses.

[Bull. Agr. Chem. Soc. Japan Vol. 23, No. 3, p. 222 230, 1959]

\title{
Effect of Carbon and Nitrogen Sources on the Utilization of Tyramine and Phenolsulphatase Biosynthesis by Cell Suspensions of Aerobacter aerogenes.
}

\author{
By Tokuya HARAdA \\ Institute of Scientific and Industrial Research, Osaka University \\ Received September 15, 1958
}

\begin{abstract}
Cell suspensions of Aerobacter aerogenes formed a remarkable amount of phenolsulphatase and dissimilate tyramine, provided a suitable primer was present with tyramine. As a primer, many carbon sources, especially, a mixture of amino acids could be considered useful. Several compounds such as glucose strongly inhibited not only the utilization of tyramine but also the phenolsulphatase synthesis of the cell suspensions containing tyramine and casein acid-hydrolyzate, while other compounds such as xylose either ineffective or only a little effective to both of the reactions. Therefore, the possibility that there might be a functional relationship between the ultilization of tyramine and the phenolsulphatase synthesis of Aerobacter aerogenes was considered.
\end{abstract}

From previous studies ${ }^{1,2)}$ it has been indicated that tyramine induces the phenolsulphatase (hereafter, referred to as PSase) synthesis of $A$. aerogenes and its specificity as an inducer is very high. Attempts to induce the PSase by some substrates, such as phenyl sulphate, $p$-nitrophenyl sulphate and urine-indican have resulted

1) T. Harada and F. Hattori, This Bulletin, 20, 110 (1956).

2) T. Harada, This Bulletin, 21, 267 (1957). in failure. It was also found that the effect of tyramine is observed not only in this bacterial species, but also in Salmonella schottmuelleri, species of Aspergillus, and Penicillium ${ }^{3)}$.

It therefore seems that the biochemical mechanism involved in it is worthy of further study. Such a study would lead us to find the net role of tyramine for the enzyme synthesis.

3) Uapublished data. 
First of all, it is desirable to obtain information concerning the presence or absence of the functional relationship between the utilization of tyramine by the organisms and their PS ase synthesis. Therefore, the effects of several carbon and nitrogen sources on the utilization of tyramine and PS ase biosynthesis of $A$. aerogenes were investigated.

\section{METHODS}

A. aerogenes ATCC 9621 was employed. This strain hardly produced PSase unless tyramine was supplied in the medium. The medium which was used to obtain cells, had the following composition: Bacto-casamino acids, 0.5 per cent; $\mathrm{KH}_{2} \mathrm{PO}_{4}$ and $\mathrm{K}_{2} \mathrm{HPO}_{4}$, each 0.05 per cent; $\mathrm{MgSO}_{4} \cdot 7 \mathrm{H}_{2} \mathrm{O}, 0.02$ per cent; $\mathrm{MnSO}_{4} \cdot 4 \mathrm{H}_{2} \mathrm{O}$, $\mathrm{FeSO}_{4} \cdot 7 \mathrm{H}_{2} \mathrm{O}$, and $\mathrm{NaCl}$, each 0.001 per cent; $\mathrm{pH} 7.2$. Resting cell suspensions were obtained by growing $A$. aerogenes, strain 9621 ATCC, in this medium at $34^{\circ} \mathrm{C}$ for 48 hours, using a one per cent inoculumn from a yeast extract broth culture. Harvesting was accomplished by centrifugation, the cells were washed thrice with distilled water and the final cell suspension was adjusted to the concentration of $5 \mathrm{mg}$ dry cell material per one $\mathrm{ml}$ with $0.2 \mathrm{M}$ phosphate buffer, $\mathrm{pH} 7.2$.

To test the effects of carbon and nitrogen sources on the utilization of tyramine and PSase induction, $4 \mathrm{ml}$ of each test solution containing the compounds to be tested and $10^{-2} \mathrm{M}$ tyramine as an inducer were added into $4 \mathrm{ml}$ of this cell suspension and the mixture aerated on a reciprocal shaker at $34^{\circ} \mathrm{C}$. A quantity of $0.5 \mathrm{ml}$ of the mixture was pipetted at appropriate intervals and $3 \mathrm{ml}$ of distilled water was added. Immediately the cells were centrifuged at about $5^{\circ} \mathrm{C}$. The supernatant was tested to determine the amount of tyramine as mentioned below. The cells, thus obtained were suspended into one $\mathrm{ml}$ of water and this suspension was transferred to a graduated test tube already containing $2 \mathrm{ml}$ of acetate buffer, $\mathrm{pH} 7.2$ and one $\mathrm{ml}$ of $0.005 \mathrm{M}$-nitrophenyl sulphate solution which had been preliminary incubated at $34^{\circ} \mathrm{C}$ for five minutes. The content of the tube was immediately brought to a final volume of $5 \mathrm{ml}$ with distilled water and was incubated further under occational shaking for 10 minutes at $34^{\circ} \mathrm{C}$. The amount of $p$ nitrophenol produced by the enzymatic action was estimated according to the method already mentioned ${ }^{(4)}$. PSase activity was expressed in units of optical density. This was determined by a Kotaki AKA photoelectric

4) T. Harada and K. Kono, J. Agr. chem. Soc. Japan, 28, 608 1954). colorimeter using a S-43 filter and a cell of $0.5 \mathrm{~cm}$ in width.

Two $\mathrm{ml}$ of each supernatant obtained from the induction mixture, was pipetted and $0.5 \mathrm{ml}$ of $0.5 \mathrm{~N}$ sodium hydroxide and $3.5 \mathrm{ml}$ of distilled water were added to this solution. The absorption of these solutions at wavelength of $294 \mathrm{~m} \mu$ was determined by a spectrophotometer (Hitachi type) in order to find the amount of remained tyramine. Such as alkaline solution of tyramine had a maximal absorption at $294 \mathrm{~m} \mu$, while a maximal absorption of a solution of tyramine hydrochloride was found at $275 \mathrm{~m} \mu$ as seen in Fig. 1.

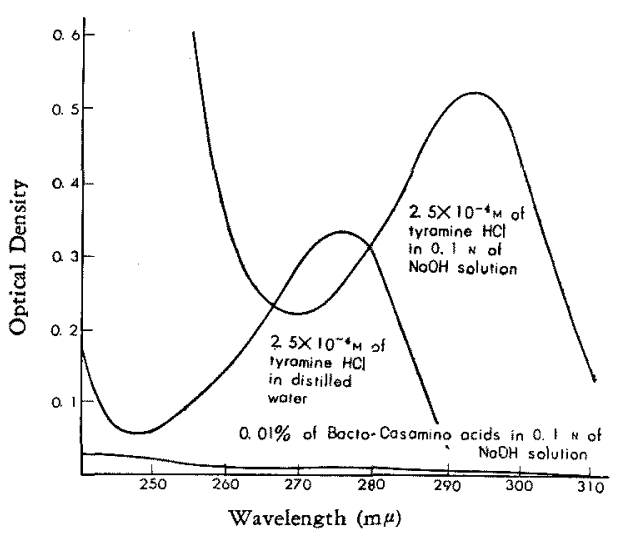

FIG. I. The Absorption Spectra of Tyramine and Bacto-Casamino Acids.

Although an alkaline solution of tyrosine also showed a maximal absorption in the neighborhood of $294 \mathrm{~m} \mu$, the amount of tyrosine contained in added 0.2 per cent casein acidhylrolyzate was so small that its absorption was too weak to be recognized in comparison with that of added $5 \times 10^{-3} \mathrm{M}$ tyramine (Fig. 1). Paper chromatographic studies indicated tyramine being utilized as no compound having the construction of phenol scemed to be accumulated in the incubation mixture.

Turbidity of the induction mixture was determined by a Kotaki AKA photoelectric colorimeter using a $\mathrm{S}=$ 66 filter. As casein acidhydrolyzate Bacto-casamino acids were used.

\section{RESULTS}

Effect of Nitrogen Sources on the Utilization of Tyramine and PSase Biosynthesis.

The cell suspensions prepared from the medium without tyramine as described in the experimental method, showed no PSase activity and could 


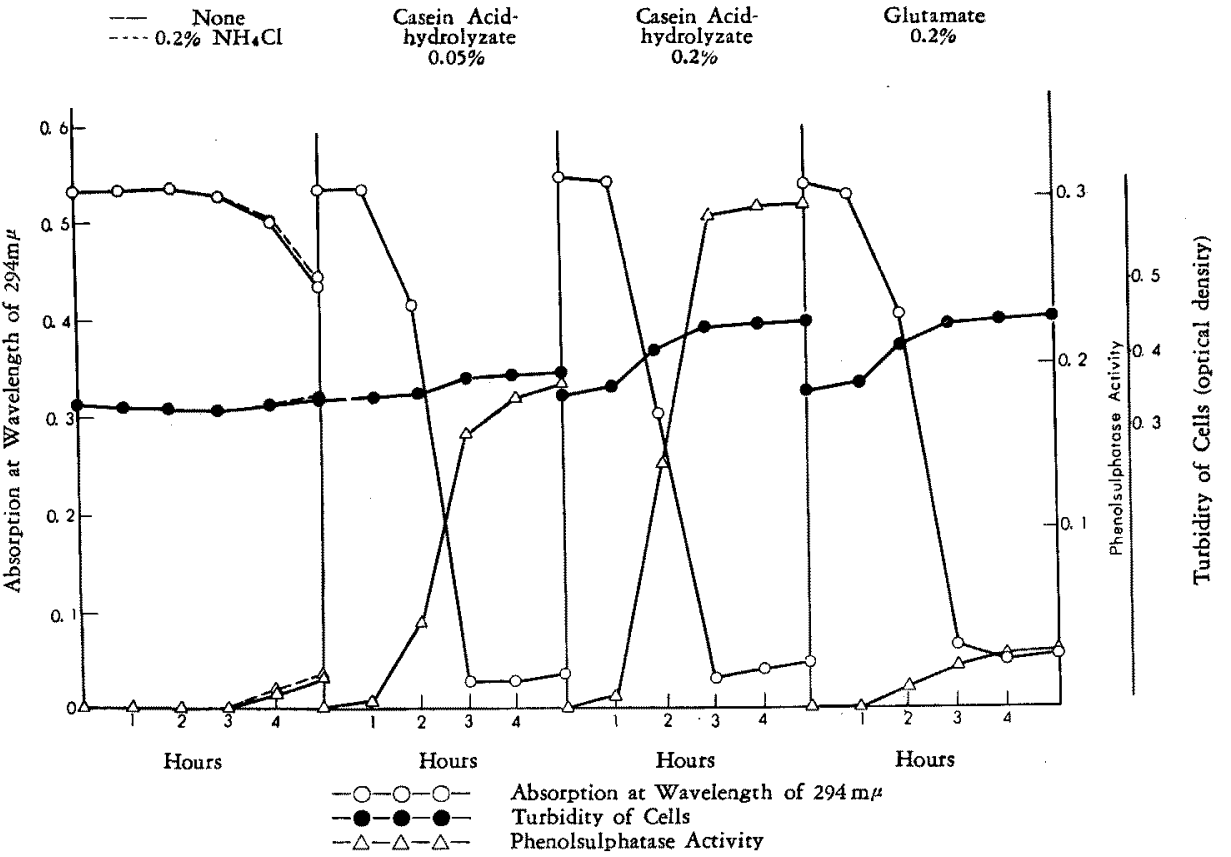

FIG. 2. The Effect of Nitrogen Sources on the Utilization of Tyramine and the Phenolsulphatase Synthesis by Aerobacter aerogenes 9621 .

not metabolize tyramine immediately. Attempts were made to test the effect of several nitrogen compounds on the utilization of tyramine and the induction of PSase synthesis in them by shaking these cells with $5 \times 10^{-3} \mathrm{M}$ tyramine. This concentration of tyramine was shown to be optimal for PSase synthesis ${ }^{(2)}$. The results of a typical experiment are illustrated in Fig. 2. Decrease of tyramine, activity of PSase and growth of cells were plotted against time of incubation. With tyramine alone or tyramine plus ammonium chloride, both the utilization of tyramine and the PSase biosynthesis were very weak. In these cases, a very slight growth of cells was observed. These results indicated that ammonium chloride was unable to replace casein acidhydrolyzate. It seems very difficult for cell suspensions of A. aerogenes, to metabolize tyramine when it was supplied alone or even in the presence of inorganic ammonium salts, although considerable PSase activity had been obtained in tyramine medium for a long incubation period as previously reported. ${ }^{(2)}$ When casein acidhydrolyzate, as the nitrogen source, was supplied with tyramine, PSase activity developed in response to the utilization of tyramine. The development of PSase activity was concerned with the concentration of casein acidhydrolyzate. When casein acidhydrolyzate was added to a 0.2 per cent concentration, a higher PSase activity developed, and tyramine was metabolized faster than in the case of 0.05 per cent concentration. But when it was increased to levels exceeding 0.2 per cent, both the utilization of tyramine and PSase synthesis were depressed.

The absorption spectra of the incubation mixture in alkaline state, which had been incubated with 0.2 per cent casein acidhydrolyzate and $5 \times 10^{-3} \mathrm{M}$ tyramine and were pipetted in time intervals of zero to 5 hours, are shown in Fig. 3. The typical absorption curve of tyra- 


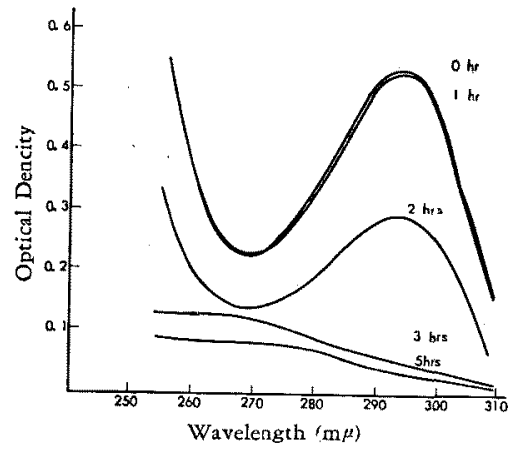

FIG. 3. The Absorption Spectra of Mixture Solution Containing Tyramine and Casein Acidhydrolyzate in Various Incubation Times.

mine (or phenol) disappeared after 3 hours, and a broad absorption curve with low optical density appeared and seemed to increase its density. From this figure and also from the results of the paper chromatographic test of the incubation mixture, it is obvious that no phenolic compound was derived from tyramine, though some substances are accumulated in the incubation mixture. The latter broad absorption curve was not due to some compounds contained in casein acidhydrolyzate before incubation, since the absorption of 0.2 per cent casein acidhydrolyzate was very weak as shown in Fig. 1. When 0.2 per cent glutamate was supplied, instead of casein acidhydrolyzate, with tyramine, a different figure was obtained as shown in the right column of Fig. 2. Although tyramine was utilized rapidly as in the case of the addition of 0.05 per cent casein acidhydrolyzate, PSase activity developed very slightly. Therefore it seemed that a mixture of amino acids would be necessary for both the rapid utilization of tyramine and the large PSase synthesis in addition to tyramine. Then the question arose, whether the organism could utilize amino acids as nitrogen sources as well as carbon and energy sources.

Effect of Carbon Sources on the Utilization of Tyramine and the PSase Biosynthesis.

Experiments were next carried out in order to find out the effect of carbon sources on the utilization of tyramine and PSase synthesis when carbon compounds were supplied with $5 \times 10^{-8} \mathrm{M}$ tyramine and 0.2 per cent casein acidhydrolyzate. Table 1 shows the PSase activity of cells, the decrease of the absorption at the wavelength of $294 \mathrm{~m} \mu$ and the growth of cells after 3 hours' incubation in various incubation mixtures.

The compounds tested as carbon sources might be divided into two groups though the effect gradually changed from one extreme (xylose) to another (rhamonse). One group (xylose etc.) was ineffective, slightly or rather moderately effective to the rapid utilization of tyramine and higher production of PSase caused by tyramine and casin acidhydrolyzate, while another group (glucose etc.) was remarkably inhibitory against both of these reactions, though the organism grew well in all of the cases. Xylose, glutamate, $\alpha$-ketoglutarate, citrate, mannose, lactose, glycerol and inositol might be included in the former group, and fructose, gluconate, glucose, L-arabinose, galactose, mannitol and rhamnose might be considered as the latter group. D-Arabinose was unable to affect any reaction since this compound could not be utilized by the organism. These observed effects could not easily be correlated with any obvious chemical features of the compounds tested. However, this phenomena could be reasonably explained by "Diauxie" "). Magasanik" has indicated that the amount of L-histidine required to support the growth of a histidine less mutant of $A$. aerogenes depends on the nature of the major carbon source of the medium and that the carbon sources can be divided into two groups. And also, the glucose group has been shown to act inhibitor against the dissimilation of histidine. The compounds used in their experiments acted in a similar fashion to the utilization of tyramine and the PSase synthesis by the authors' experiments, with the exception of fructose and rhamnose. Our earlier studies with

5) J. Monod, Researches sur la croîssance des cultures bacteriennes, Paris (1942).

6) B. Magasanik, J. Biol. Cbem., 213, 557 (1955). 
culture medium showed that glucose inhibited the formation of PSase by this strain. A similar finding had been made by Whitehead et al. ${ }^{(7)}$. They showed that no PSase could be detected in any of the tested strains of Salmonella and some strains of mycobacteria being considered to be PSase positive strains, when 0.5 per cent glucose was contained in culture medium. The reason for these inhibitions might be explained in various incubation periods with and without casein acidhydrolyzate.

As seen in Fig. 4, a remarkable delay of the inducing time for the utilization of tyramine and PSase synthesis was observed in the incubation mixture supplied with glucose both in the presence and absence of casein acidhydrolyzate in comparison with that supplied with xylose. Further, in the absence of casein acidhydrolyzate,

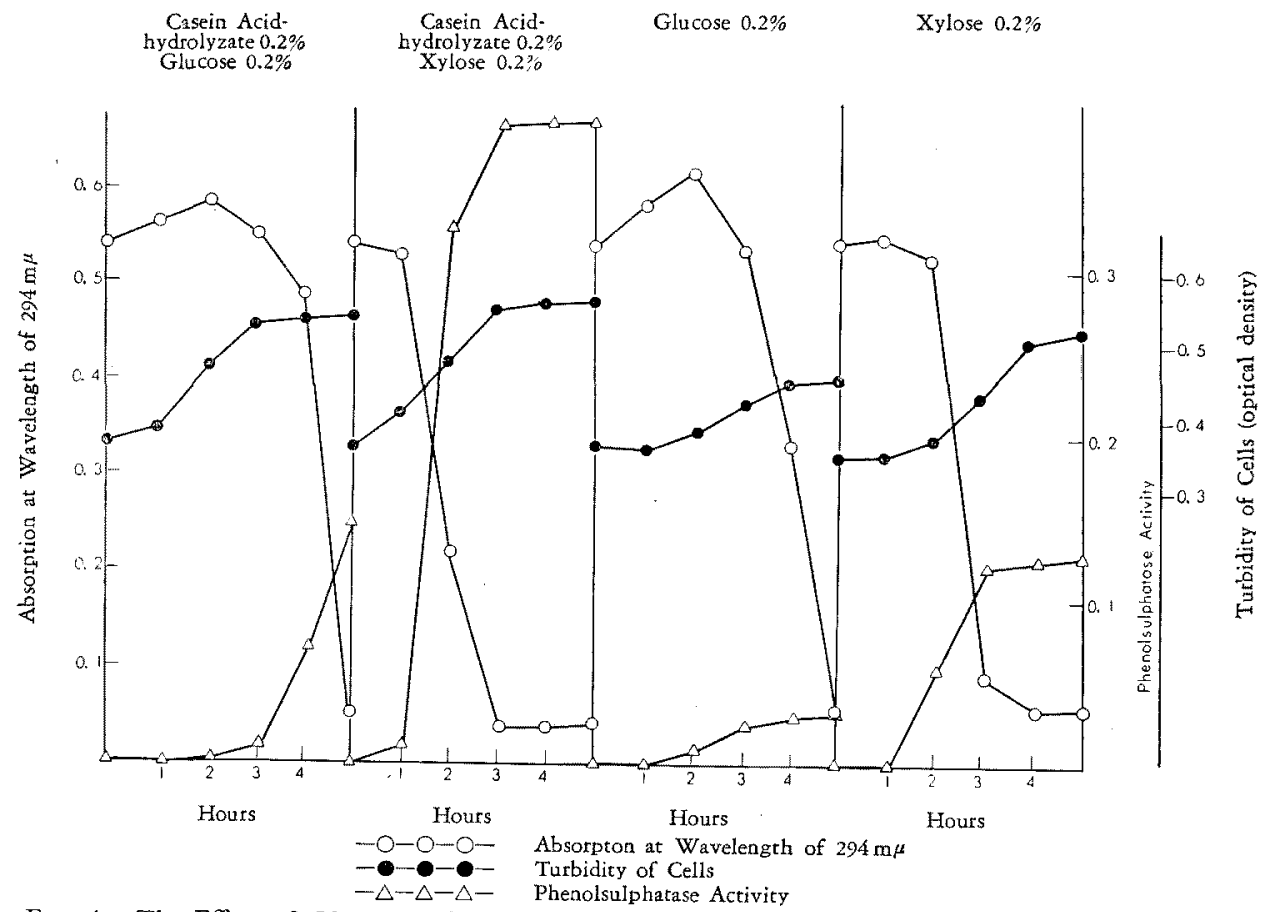

FIG. 4. The Effect of Glucose and Xylose on the Utilization of Tyramine and the Phenolsulphatase Synthesis in the Presence and Absence of Casein Acidhydrolyzate by Aerobacter aerogenes 9621.

by the same mechanism.

From these results it was found that any substance inhibiting the dissimilation of tyramine, also inhibited PSase synthesis.

In order to clarify their relations more clearly, the effects of glucose and xylose, as the representatives of two groups on the utilization of tyramine and PSase synthesis, were investigated

7) J. E. M. Whitehead, A. R. Morrison and L. Young, Bivchem. J., 51, 585 (1952). growth of bacteria when supplied with glucose, was shown to be much poorer than in the case of xylose. These facts indicated that in the presence of casein acidhydrolyzate, tyramine might be utilized after the disappearance of glucose, this being reasonably explained by the "Diauxie" phenomenon and that in the absence of casein acidhydrolyzate glucose is less effective as a primer for the utilization of tyramine than xylose. 
Table I. The EFfect of Various Carbon Sources on the Utilization of Tyramine and the PSase Synthesis in the Presence of CASEIN ACIDHYDROLYZATE BY Aerobacter aerogenes.

\begin{tabular}{lccc}
\multicolumn{1}{c}{ Compounds } & Phenolsulphatase Activity & $\begin{array}{c}\text { Decrease of Absorption } \\
\text { at }\end{array}$ & $\begin{array}{c}\text { Turbidity of Cells } \\
\text { (optical density) }\end{array}$ \\
Xylose & 0.38 & 0.50 & 0.20 \\
Glutamate & 0.30 & 0.50 & 0.18 \\
$\alpha$-Ketoglutarate & 0.30 & 0.50 & 0.14 \\
D-Arabinose & 0.31 & 0.50 & 0.10 \\
None & 0.30 & 0.50 & 0.10 \\
Citrate & 0.22 & 0.33 & 0.13 \\
Mannose & 0.12 & 0.12 & 0.14 \\
Lactate & 0.09 & 0.19 & 0.12 \\
Glycerol & 0.09 & 0.15 & 0.12 \\
Inositol & 0.09 & 0.20 & 0.15 \\
Fructose & 0.045 & -0.09 & 0.13 \\
Gluconate & 0.04 & 0.04 & 0.11 \\
Glucose & 0.025 & -0.25 & 0.17 \\
L-Arabinose & 0.02 & -0.04 & 0.16 \\
Galactose & 0.008 & -0.027 & 0.14 \\
Mannitol & 0.007 & -0.043 & 0.08 \\
Rhaminose & 0.005 & -0.025 & 0.14
\end{tabular}

In the case of glucose, the absorption optical density at the wavelength of $294 \mathrm{~m} \mu$ increased after incubation for one or two hours. However, this increase of absorption was found to appear at wavelength ranging from $250 \mathrm{~m} \mu$ to $310 \mathrm{~m} \mu$, approximately at in the same rate. Therefore, this increase might be due to the appearance of some compound derived mainly from glucose under these conditions. On addition of each member of the glucose group, a similar attitude was observed (Table I). In the case of xylose group members, no such increase of absorption occurred. This may be explained that tyramine might be dissimilated as fast as xylose or other compounds.

It was also demonstrated that PSase activity becomes slightly higher, when xylose is added in the incubation mixture which consisted of tyramine and casein acidhydrolyzate. The reason for this has not yet been clarified. When xylose was added with tyramine, but without casein acidhydrolyzate, the dissimilation of tyramine was delayed to a certain extent and the activity of PSase was about 40 per cent in comparison with the case of both tyramine and casein- hydrolyzate.

From the comparison of experimental data obtained from a series of combination of elementary compounds, such as tyramine only, tyramine plus xylose, tyramine plus glucose, tyramine plus glutamate, tyramine plus casein acidhydrolyzate, the following conclusion was made. The carbon of tyramine can be utilized easily provided a suitable primary carbon source is supplied. Mixture of amino acids such as casein acidhydrolyzate is the best for both the utilization of tyramine and PSase synthesis. The formation of PSase by this organism starts with the utilization of tyramine. A. aerogenes seems to have a weak or either no activity in utilizing tyramine without a primary carbon or "primer".

PSase Synthesis by Tyramine Adapted Cells.

In order to ascertain whether external substances are necessary for PSase synthesis by tyramine adapted cells, first of all various concentrations of casein acidhydrolyzate were tested for their effect in supporting the utilization of tyramine and PSase synthesis. Tyramine adapted cells were obtained by the following 


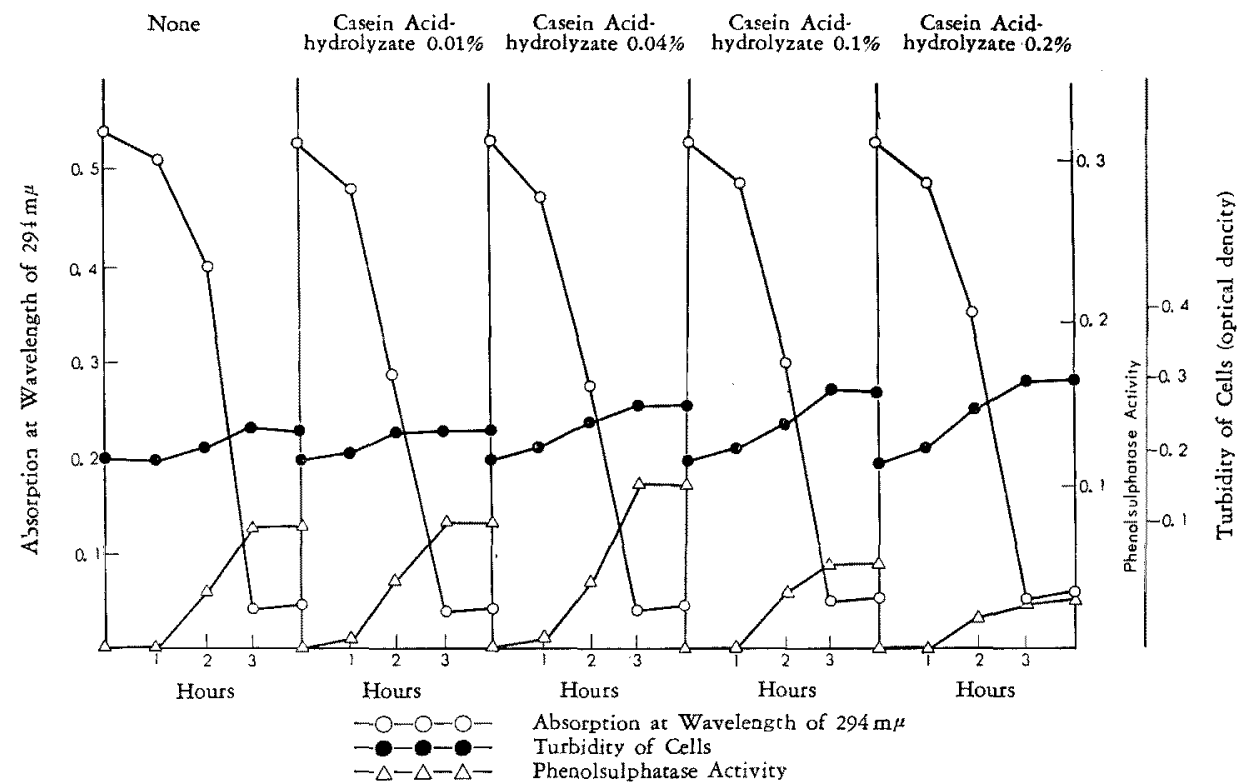

FiG. 5. The Effect of Casein Acidhyrolyzate on the Utilization of Tyramine and the PSase Synthesis by Tyramine Adapted Cells of Aerobacter aerogenes 9621 .

Table II. The EfFect of Some Carbon Sources on the Utilization of Tyramine and the PSASE SYNTHESIS BY TYRAMINE ADAPTED Cells OF Aerobacter aerogenes.

Compounds

Concentration (per cent)

None

Casein Acidhydrolyzate $\left\{\begin{array}{l}0.01 \\ 0.04 \\ 0.1 \\ 0.2\end{array}\right.$
Glutamate
Xylose $\left\{\begin{array}{l}0.01 \\ 0.04 \\ 0.1 \\ 0.2 \\ 0.01 \\ 0.04 \\ 0.1 \\ 0.2 \\ 0.01 \\ 0.04 \\ 0.1 \\ 0.2\end{array}\right.$

method: Cells harvested as above mentioned from the culture medium, were washed and suspended in a solution containing $5 \times 10^{-3} \mathrm{M}$
Phenolsulphatase
Activity

0.070

0.075

0.100

0.060

0.025

0.035

0.025

0.020

0.015

0.060

0.055

0.040

0.035

0.060

0.055

0.030

0.025
Decrease of Absorption at Wavelength of $294 \mathrm{~m} / \mu$
0.49
0.49
0.48
0.48
0.48
0.48
0.47
0.47
0.47
0.49
0.48
0.48
0.48
0.49
0.49
0.31
0.23

tyramine and 0.2 per cent glutamate with phosphate buffer. The incubation mixture was aerated for one hour and a quater. The cells were 
devoid of recognizable PSase activity. Then, the cells were harvested and washed twice with distilled water. The adapted cells were subsequently aerated with $5 \times 10^{-3} \mathrm{M}$ tyramine and varying concentrations of casein acidhydrolyzate.

Fig. 5 indicates that although a small amount of PSase was synthesised without casein acidhydrolyzate, the activity of PSase was increased by the addition of 0.01 or 0.04 per cent casein acidhydrolyzate. However, in the case of a larger amount of casein acidhydrolyzate $(0.1$ or 0.2 per cent), a considerable inhibition against the PSase synthesis was observed. When xylose, glucose or glutamate was added in varying concentrations instead of casein acidhydrolyzate, inhibition against PSase synthesis occurred in every case, even in a very dilute concentration. From these experiments, it was apparent that a small amount of external amino acids promoted the synthesis of PSase as well as the utilization of tyramine by the tyramine adapted cells. Other carbon sources tested were not considered necessary for the utilization of tyramine and were rather inhibitory against the PSase synthesis.

\section{DISCUSSION}

Previous studies indicated that tyramine induced the PSase synthesis of $A$. aerogenes and its specificity as an inducer was very high. More recently, working with Salm. schottmuelleri, the author also observed a remarkable effect of tyramine on the PSase synthesis of this microorganism. Both species of bacteria were capable of dissimilating tyramine. Furthermore, in the culture of many species of Aspergillus and Penicillium similar effects of tyramine were found although a considerable amount of PSase developed even when tyramine was not supplied. It was also confirmed that these molds could dissimilate tyramine. On the other hand, in many species of Rhizopus having an ability to form PSase but incapable of dissimilating tyramine, no effect of tyramine has yet been observed. Such an effect was not found in Str. alcalophilus, the principal microorganism which caused the coloration of lignin in putryfying urine $^{8,9)}$. A study based on this mold will be published in the future. Accordingly, it seems probable that tyramine would affect PSase synthesis only by action of the microorganisms having capacity of dissimilating tyramine. These findings have lead the author to investigate whether or not oxidative dissilation of tyramine is always necessary for the development of PSase by many microorganism species.

In the present study, the relationship between the utilization of tyramine and the PSase synthesis was investigated by cell suspensions of $A$. aerogenes when various substances were supplied in addition to tyramine. From experiments with this organism, it was found that tyramine could hardly be utilized and a very small amount of PSase was formed unless amino acids or other carbon sources were supplied. PSase was synthesized in response to the utilization of tyramine which was caused by the addition of appropriate substances with tyramine. When various carbon sources were supplied with tyramine and casein acidhydrolyzate, several compounds such as glucose inhibited not only the utilization of tyramine but also PSase synthesis, while other compounds such as xylose were ineffective or only a little effective to both reactions. Therefore, this possibility was considered that the oxidative dissimilation of tyramine might be necessary for PSase synthesis. However the fact whether any metabolism of tyramine is concerned with PSase synthesis has not been ascertained in this study.

In any case, PSase could develop only when tyramine was metabolized in the case of this strain, although great differences in PSase activity developed by the effect of various substances which were added with tyramine were recognized. Among many of these compounds, casein acidhydrolyzate proved to be most effective for PSase synthesis in both cases of tyramineadapted and nonadapted cells. These facts indicated that amino acids, as well as serving as

8) T. Harada, J. Agr. Chem. Sac. Japan, 26, 95 (1952).

9) T. Harada, J. Agr. Chem. Soc. Japan, 29, 516 (1955). 
nitrogen sources, might play a role as sparkers or primers of tyramine dissimilation for the enzyme synthesis.
Acknowledgement: The author wishes to express his sincere thanks to Prof. Z. Nikuni for his kind advice and suggestions in the course of this work.

[Bull. Agr. Chem. Soc. Japan, Vol. 23, No. 3, p. 230 233 1959]

\title{
Studies on Phenolic Lactones
}

\section{Part II. A New Synthetic Method of Isohibalactone}

\author{
By Kyôhei Yamashita and Masanao Matsui \\ Faculty of Agriculture, University of Tokyo \\ Received September 16, 1958
}

\begin{abstract}
Isohibalactone, the geometric isomer of hibalactone, was synthesized by the following route. Piperonylsuccinic acid anhydride was converted into thioethyl methyl ester ans was reduced to piperonylbutyrolactone by Raney nickel catalyst. Piperonylbutyrolactone was also prepared from piperonylsuccinic anhydride by the reduction with amalgamated aluminum. Condensation of piperonal with the lactone in the presence of potassium amide afforded $\alpha$-(3,4-methylenedioxyphenyl-hydroxymethyl)- $\beta$-(3,4-methylenedioxybenzyl)-butyrolactone, m.p. $151 \sim 2^{\circ} \mathrm{C}$. Dehydration of the hydroxylactone with $p$-toluenesulfonic acid gave isohibalactone, m.p. $156 \sim 6.5^{\circ} \mathrm{C}$.
\end{abstract}

In the previous paper ${ }^{12}$ the authors reported the synthesis of $\alpha$-piperonylidene- $\beta$-piperonylbutyrolactone from the corresponding succinic<smiles>O=C1OOCCC1CC1CCC2OCOC2C1</smiles>

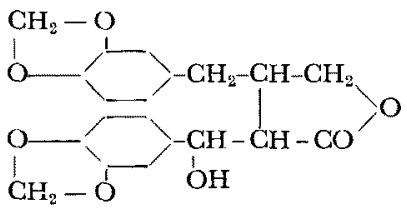

(II)

acid. But the product was not identical with hibalactone in its infrared spectra and the authors designated it as isohibalactone. As was

\footnotetext{
1) K. Yamashita and M. Matsui, This Bulletin, 22, 227 (1958),
}

described there, during the reduction of thioethylester with Raney nickel catalyst most of the double bond was also hydrogenated and the<smiles>CC(C)CC1CCC2OCOC2CC1</smiles>

(I)

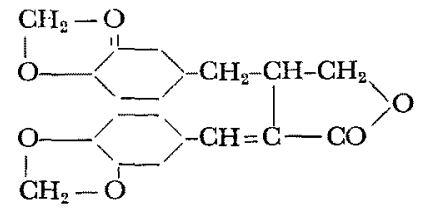

(III)

yield of unsaturated lactone was rather poor. Therefore the authors deviced an another route to $\alpha$-piperonylidene- $\beta$-piperonyl-butyrolactone.

For the synthesis of $\beta$-piperonylbutyrolactone 